\title{
Arousal from sleep: The effects of the rise-time of auditory stimuli
}

\author{
T. E. LeVERE, N. DAVIS, JEANNE MILLS, ELLIOTT H. BERGER, and WILLIAM F. REITER \\ North Carolina State University, Raleigh, North Carolina 27607
}

\begin{abstract}
The present research involves the methodological strategy of investigating sleep by determining an individual's reaction to extrinsic stimulation. Particularly, the investigation was concerned with whether or not the onset character of an auditory stimulus was an important parameter in precipitating changes in the pattern of electroencephalographic activity occurring during sleep. The results indicated that stimulus onset is an important parameter but only during sleep characterized by slow-wave electroencephalographic activity. In this respect, the effects of stimulus onset are similar to the effects of psychological loudness. The results are also discussed in relation to the effects of stimulus onset when awake individuals are exposed to auditory stimuli, and, again similar to psychological loudness, the data from awake individuals is not predictive of an individual's reaction during sleep.
\end{abstract}

Defining the nature of a particular psychophysical condition involves, at least in part, specifying how an individual reacts to stimulation when the particular psychophysical condition exists. Thus, for example, the state produced by a particular pharmacological agent may be investigated by observing an individual's response to extrinsic stimulation while under the systemic influence of the assimilated drug. Similarly, the conditions produced by neurological dysfunction may be assessed by determining how an individual responds when certain areas of the brain are rendered inactive. With respect to the investigation of sleep, a similar methodology has been employed with some success.

Early research studying an individual's reaction to stimulation during sleep was principally concerned with determining depth of sleep as indicated by the intensity of extrinsic stimulation required to produce behavioral awakening (see, for example, reviews by Jones, 1964; Kleitman, 1963). While the specific question of depth of sleep has been put aside, principally because of a lack of correlation between concomitant indices of sleep (Goodenough, Lewis, Shapiro, Jaret, \& Sleser, 1965; Rechtschaffen, Hauri, \& Zeitlin, 1966), the general methodology has survived. For example, Williams, Hammack, Daly, Dement, and Lubin (1964) measured an individual's reaction to extrinsic auditory stimulation to investi-

This research was supported by the National Aeronautics and Space Administration Research Grant NGL 34-002-095. T. E. LeVere, N. Davis, and Jeanne Mills are members of the Neuropsychology Laboratory, Department of Psychology, and Elliott H. Berger and William F. Reiter are members of the Center for Acoustical Studies, Department of Mechanical and Aerospace Engineering. The authors wish to thank D. Miller for her assistance in preparing this report. Requests for reprints should be directed to: T. E. LeVere, Neuropsychology Laboratory, Department of Psychology, North Carolina State University, Raleigh, North Carolina 27607. gate the effects of sleep deprivation. Other research has concentrated on the averaged neural response evoked by auditory stimulation to further define the parameters of different sleep stages (Amadeo \& Shagass, 1973; Mendel \& Goldstein, 1971; Weitzman \& Kreman, 1965; Williams, Tepas, \& Morlock, 1962). Moreover, questions involving such events as habituation, with its connotation of learning, have also been approached through the tabulation of an individual's reaction to extrinsic stimulation during sleep (Firth, 1973; Johnson \& Lubin, 1967; Sokolov \& Paramonova, 1961). While the above researchers have been principally, although certainly not exclusively, concerned with the effects of relatively constant extrinsic stimulus presentations during different types of sleep, other research has taken a somewhat different empirical tack and investigated the sleeping individual's reaction to varying stimulus conditions. For example, the arousal produced by stimuli differing in cognitive value (familiarity) has been investigated by Zung and Wilson (1961). And, in a somewhat more quantitative vein, LeVere, Bartus, and Hart (1973) and LeVere, Morlock, Thomas, and Hart (1974) measured the arousal produced by auditory stimuli varying in frequency but equated for subjective loudness. While each of the above researches are clearly unique in detail, all share the underlying theme of questioning the nature of sleep by quantifying an individual's reaction to stimulation during this psychophysical state. The presently reported research is in this general empirical tradition. Specifically, the research which is described below is an attempt to determine whether the onset characteristic of an extrinsic auditory stimulus would influence the individual's reaction to that stimulus. In particular, the question was whether or not an auditory stimulus having a rapid, virtually instantaneous, onset might produce a greater change 
in the pattern of a sleeping individual's electroencephalographic activity as compared to an auditory stimulus similar in frequency and intensity but having a more gradual onset. Summarily, the answer appears to be yes, but not always.

\section{METHOD}

\section{Subjects}

The subjects for this research were male college student volunteers between the ages of 19 and 23 years (mean $=20.3$ ). The subjects were selected from a larger population of volunteers to form, insofar as possible, a homogeneous group. The selection criteria involved the individual's subjective estimate of his normal sleep routine and included such considerations as: (1) the usual time he retired at night and arose in the morning, (2) the amount of time normally required to fall asleep, (3) whether he considered himself a heavy or light sleeper, and (4) whether he had difficulty falling asleep in strange environments such as hotels, motels, and the like. None of the subjects was currently under a physician's care or taking any medication which might interfere with his sleep. With the exception of sleeping within the laboratory, all of the subjects were instructed to maintain their normal daily routines, excluding, of course, the use of alcohol or other narcotics during their participation in the experiment.

\section{Apparatus}

The general apparatus used in the present research has been detailed by LeVere et al. $(1973,1974)$. Briefly, the subjects slept each night in the laboratory mockup bedroom $(12 \times 12 \times 8 \mathrm{ft})$ constructed in accord with standard building practices. This bedroom was located within a larger laboratory area where the ambient noise level during the night was less than $45 \mathrm{~dB}$, as measured with the A-weighted scale (General Radio Type 1565-A). For numerous reasons, but principally acoustical, the bedroom was furnished with a carpet, a small dresser, and two chairs in addition to the auditory speaker system which was located some $14 \mathrm{ft}$ from the subject's bed.

The subject's eletroencephalographic (EEG) activity was used to both distinguish certain types of sleep for stimulus presentations and, additionally, quantify the subject's reaction (arousal) to the stimulus presentations. The EEG activity was recorded monopolarly with a 9-mm silver cup electrode (Grass Instrument Company No. E5S) attached to the P3 location (International 10-20 System) and referenced to the ipsilateral ear lobe. A similar electrode attached to the outer canthus of the ipsilateral eye and referenced to the same ear lobe was used to record the electrooculogram (EOG). A final electrode was attached to the subject's neck for grounding purposes. It is of course recognized that this limited electrode array deviates from that suggested by Rechtschaffen and Kales (1968) for the evaluation of sleep stages by visual inspection of polygraph records. However, the present computer evaluation of the subject's electroencephalographic activity was principally concerned with the detection of sleep characterized by low voltage mixed frequency EEG activity without rapid eye movements and sleep characterized by slow-wave EEG activity and not any finer subdivisions within these categories. Regarding this, the more limited, but more convenient, electrode arrrangement described above was opted as being more appropriate.

The EEG and EOG activity was amplified by a Grass Model 79 polygraph and band passed filtered between $1.0 \mathrm{~Hz}$ and $0.1 \mathrm{kHz}$ with the filte's available on the instrument. The amplified analogue signals were then band passed filtered again with a Rockland Model 1022F filter $(1.0$ and $35 \mathrm{~Hz})$ and passed directly to a Digital Equipment Corporation PDP-12A computer for on-line analysis. The PDP-12A computer was programmed in accord with our standardized Zero-Crossings frequency analysis
(LeVere et al., 1973, 1974). Basically, this procedure is a program which determines individual frequency components on the basis of the time that elapses between successive crossings of zero potential by the digitized analogue signal. The program utilizes successive $40-\mathrm{sec}$ analysis epochs and reduces the frequency data within each $40-\mathrm{sec}$ epoch by accumulating similar frequencies according to the classical band widths of delta $(0-3 \mathrm{~Hz})$, theta (4-7 Hz), alpha (8-12 Hz), and beta $(13-35 \mathrm{~Hz})$, as well as the total number of half-wave occurrences. Each of the successive 40-sec analysis epochs is separated by a $20-\mathrm{sec}$ interepoch interval to allow for on-line data printout. As with previous research utilizing this methodology, the four frequency bands were principally used to define the electroencephalographic character of an individual's sleep for stimulus presentations. The major dependent measure for determining an individual's reaction to a given stimulus presentation was the total number of half-waves which occurred during a given epoch, i.e., the overall level of cortical desynchronization. This particular dependent variable was used for two reasons. First, there is a relatively clear direct relationship between cortical desynchronization (increases in the total number of half-wave occurrences) and arousal (Adrian \& Matthews, 1934; Moruzzi \& Magoun, 1949; Rheinberger \& Jasper, 1937; Sokolov, 1960), and secondly, the total number of half-waves is a dependent measure which allows analysis by standard parametric statistical procedures.

Two auditory stimuli were used in the present experiment. The stimuli were identical except for their onset and offset characteristics and were generated by filtering out all frequencies of a random noise source save a one-third octave band centered on the frequency of $125 \mathrm{~Hz}$. Both stimuli had a duration of $15 \mathrm{sec}$ at their peak intensity level of $80 \mathrm{~dB}$ measured with the A-weighted network. One of the auditory stimuli, the fast-rise stimulus, reached this maximum intensity level instantaneously or at least within the limits of mechanical relay operation. As measured with an oscilloscope, the closure of this relay averaged $3.24 \mathrm{msec}$ with a standard error of $.07 \mathrm{msec}$ over 25 measurements. Figure 1 shows the power spectrum of this stimulus as computed during its onset (lower part of figure) and after it had stabilized at its maximum intensity (upper part of figure). The similarity of these two plots indicates that the rapid onset of the stimulus did not significantly change the frequency characteristics of this stimulus. The second stimulus, the slow-rise stimulus, approached the maximum intensity in a gradual, but linear, fashion over a period of approximately $7.5 \mathrm{sec}$. This gradual onset was accomplished by mechanically coupling the gain control of the amplifying system to a synchronous motor, which, when energized, simply turned the gain control to the preset maximum of $80 \mathrm{~dB}(\mathrm{~A})$. When the stimulus had obtained its maximum intensity, it remained at this level for the standard 15 -sec duration, after which the motor was reversed and energized to decrease the intensity of the stimulus to zero. Acoustical measurements taken as described in Figure 1 indicated that both stimuli had identical power spectra and that the rise and fall ramps of the slow-rise stimuli were linear.

\section{Procedure}

Each subject was required to come to the laboratory for 3 successive nights, with 1 of the nights designated as a control night, where the subject's sleep was undisturbed, and the other 2 nights designated as stimulus nights, where the subject was exposed to 24 presentations of either the fast-rise stimulus or 24 presentations of the slow-rise stimulus. The sequential order of the two stimulus nights and the single control night was balanced across the nine subjects to control for any order and/ or carryover effects which could potentially bias the experimental conditions. For data acquisition purposes, the first $6 \mathrm{~h}$ of an individual's sleep were used, and this period was divided into 36040 -sec analysis epochs, each of which was separated by the standard 20-sec interepoch interval.

During the stimulus nights, stimulus presentations were in accord with a schedule programmed into the computer which 

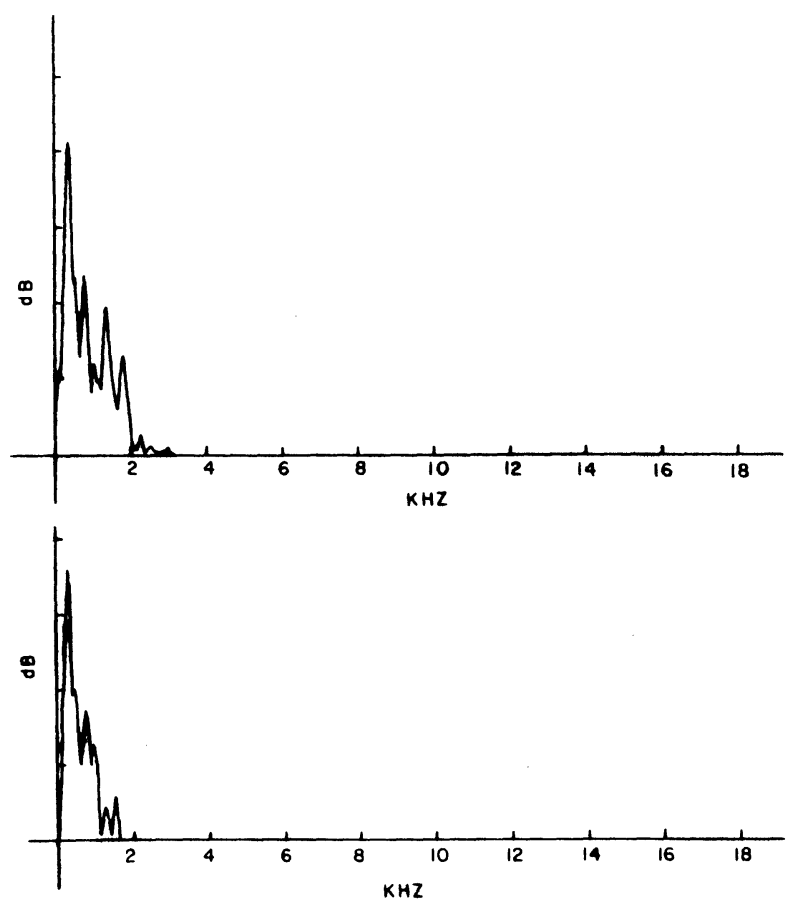

Figure 1. Power spectrum plots of the fast-rise stimulus obtained during its steady state at maximum intensity (upper portion) and during its onset (lower portion). Both plots were obtained with a Spectral Dynamics SD 301B real-time analyzer and were computed on the basis of 32.025 -sec samples taken over a total time of $.8 \mathrm{sec}$. The absissa indicates $\mathrm{dB}$ level, with distance between each mark representing $10 \mathrm{~dB}$. The ordinate gives the frequency. The overall level of the power spectrum computed during the onset is somewhat lower because of the necessity of taking some averages when the stimulus was not present in order to bracket the onset of the stimulus. However, this does not affect the relative relations between this plot and that obtained when the stimulus was at its maximum intensity. Similar spectrum computed over a more restricted frequency range $(0-k H z)$ allowed greater resolution $(15 \mathrm{~Hz})$ and produced virtually identical steady state and onset plots.

distributed the 24 stimulus presentations over the 6-h data period manner so that: (1) 12 of the presentations were to occur when the subject's EEG pattern exhibited non-REM fast-wave activity, (2) 12 of the presentations were to occur when the subject's EEG pattern exhibited slow-wave activity, and (3) no stimulus presentations were to occur if REM occupied $10 \%$ or more of either of the two immediately preceding analysis epochs. The latter restriction reflects our attempt to exclude stimulus presentations during REM sleep. The exctusion of stimutus presentations during this type of sleep was considered desirable for two reasons. First, if the subject is dreaming, it is more likely that this will occur during REM sleep, and since there exists the possibility that extrinsic stimuli may be incorporated into dream content (Freemon, 1972; Rechtschaffen et al. 1966), a subject's response may be somewhat of a definitional matter during this type of sleep. Secondly, and clearly more important, REM sleep is characterized by a very desynchronized pattern of EEG activity which would serve to place a ceiling upon our dependent measure and make an individual's reaction difficult to quantify with our procedure even should the stimulus presentation not be incorporated into dream content. It is, of course, realized that the utilization of a single electrode to signify eye movements risks the possibility of contaminating the EOG record with ongoing
EEG activity, particularly large-amplitude slow-wave EEG activity. However, this error is somewhat conservative, in the sense that it produces an overestimation of REM sleep.

The computer determination of non-REM fast-wave and slowwave sleep was accomplished similarly to previously reported procedures (LeVere et al., 1973, . 1974) by computing the time within each 40 -sec analysis epoch that was consumed by the accumulated frequencies within the categories of delta, theta, alpha, and beta. Slow-wave sleep was scored when the EEG epoch indicated $20 \%$ or more slow-wave delta activity on a time basis. On the other hand, if the delta occurred less than $20 \%$ of the time and, additionally, the alpha and beta activity were also less than $20 \%$, the subject was considered to be in fastwave sleep. Wakefulness was assumed when the EEG activity fell within neither the fast-wave sleep category nor the slowwave sleep category. Validation of these decision criteria with respect to visual analysis of the EEG polygraph record has been previously reported by LeVere et al. (1974), and indicated a significant agreement between the visual analysis and the computer analysis of the sleep polygraph records with respect to the categories of wakefulness, non-REM fast-wave sleep, and slowwave sleep.

On each night the subject slept in the laboratory, the EEG and EOG recordings were initiated immediately after the subject was settled in bed. From this point onward, the control of the experiment as well as the analysis of the data were cmpletely under computer control. The 6-h data acquisition period was started after the subject's EEG record indicated five successive 40-sec analysis epochs of sleep as described by the criterion above. The first stimulus presentation was scheduled to occur 5-min after this point in time. If there were no rapid eye movements during the two epochs immediately preceding the stimulus presentation, the computer stored the EEG data with respect to the frequency categories of delta, theta, alpha, and beta as well as the total number of half-wave occurrences as these were detected during: (1) the 40-sec analysis just preceding this stimulus presentation, (2) the 40-sec analysis epoch during which the stimulus was presented, and (3) the three 40-sec analysis epochs which succeeded the presentation of the stimulus. Control nights were programmed and analyzed in exactly the same manner as described for stimulus nights except, of course, no auditory stimuli were presented to the subject.

\section{RESULTS}

The results of this procedure may be summarized quite briefly; during sleep, the onset characteristic of an auditory stimulus would appear to be an effective parameter in determining an individual's reaction. However, there is a qualification to this rather simplistic conclusion. That is, the influence of stimulus onset or rise time is not constant during all types of sleep.

The specific data are presented in Figure 2. This figure shows that mean cortical desynchronization (mean total number of half-waves of arousal computed over all subjects) which was associated with the nocturnal auditory stimulus presentations. The data are presented relative to the 40 -sec analysis epoch just preceding the stimulus presentation, the 40 -sec analysis epoch when a stimulus occurred, and the three succeeding 40-sec analysis epochs. The figure further dissociates the two types of stimuli (fast-rise and slow-rise) and the control condition (no stimulus presentations) and then dichotomizes this data relative to sleep character- 


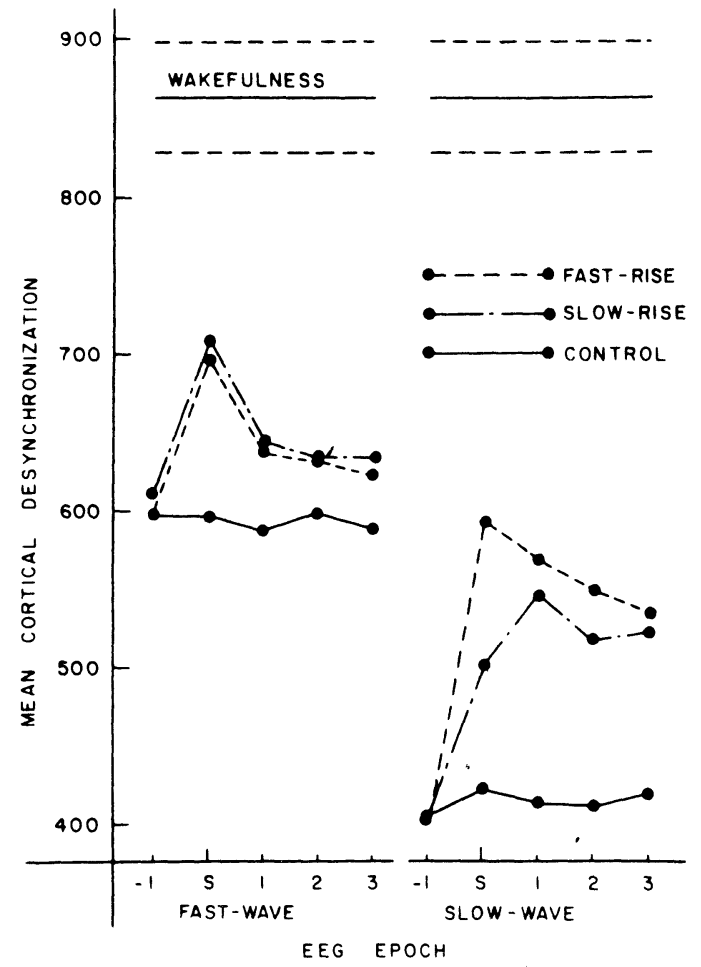

Figure 2. Mean number of half-waves (cortical desynchronization or arousal) shown in the sleeping subject's EEG record during the analysis epoch just preceding a stimulus presentation, the analysis epoch when the stimulus occurred, and the three succeeding analysis epochs. The figure summarizes the data collected over all subjects and presents separate curves for the two types of auditory stimuli and the single no-stimulus control condition. These mean data are segregated relative to sleep characterized by non-REM fast-wave EEG activity (left side of figure) and sleep characterized by slow-wave EEG activity (right side of figure). The solid horizontal line labled "wakefulness" is the mean cortical desynchronization recorded just prior to sleep onset, with the .05 confidence limits indicated by the parallel dashed lines.

ized by fast-wave EEG activity (left side of figure) and sleep characterized by slow-wave EEG activity (right side of figure). The solid line at the top of the figure is an estimate of wakefulness and is based upon the cortical desynchronization recorded during the 40 -sec analysis epoch just prior to the first of the five successive epochs indicating that the subject was asleep. The dashed horizontal lines above and below this solid line are the .05 confidence intervals associated with this mean value.

In general, Figure 2 indicates that auditory stimuli having a fast onset and auditory stimuli having a slow onset are both effective in producing some degree of arousal during sleep. However, the stimuli appear somewhat more effective during sleep characterized by slow-wave EEG activity than during sleep characterized by non-REM fast-wave EEG activity, both in terms of the amount of cortical desynchronization and its duration. Considering more closely the subject's reaction occurring during these two types of sleep, the data indicate that the two types of stimuli were essentially equally effective in producing arousal during sleep characterized by non-REM fast-wave EEG activity but were not equally effective during sleep characterized by slow-wave EEG activity. During slow-wave sleep, the auditory stimuli which had the rapid onset, that is the fast-rise stimuli, produced considerably more arousal as compared to the auditory stimuli having the more gradual onset. This result is somewhat surprising in view of the fact that both the fast-rise and the slow-rise stimuli had a duration of $15 \mathrm{sec}$ once attaining their maximum intensity level of $80 \mathrm{~dB}(\mathrm{~A})$. This then necessarily resulted in a greater amount of total energy being associated with the slowrise stimulus because of its onset and offset ramps. However, even with its greater total energy and duration, the slow-rise stimulus appeared less effective in arousing the individual.

Statistically, these results were analyzed with separate repeated measures analysis of variance applied to the data collected during the sleep characterized by nonREM fast-wave EEG activity and sleep characterized by slow-wave EEG activity. In both cases, the mean difference score was used as the dependent variable and was calculated by subtracting the cortical desynchronization occurring during the analysis epoch just preceding a stimulus presentation from: (1) the cortical desynchronization occurring during the analysis epoch when a stimulus was presented, and (2) the cortical desynchronization occurring during each of the three successive analysis epochs. The mean of these four difference scores was then calculated and used to estimate the effect of the given stimulus with the mean of similar stimuli providing the dependent measure for the Treatments by Subject's ANOVA. For sleep characterized by non-REM fast-wave EEG activity, the analysis of variance produced a significant $F$ ratio $(\mathrm{F}=19.9, \mathrm{df}=2,16, \mathrm{p}<.05)$. Individual comparison based on the calculated critical difference value (Lindquist, 1956) indicated that the arousal produced by the fast-rise auditory stimulus and the arousal produced by the slow-rise auditory stimulus were both significantly different from the control condition but not from one another (confidence level $>.05$ ). Considering the stimulus presentations during sleep characterized by slow-wave EEG activity, the overall analysis of variance was again significant $(F=37.4$, $\mathrm{df}=2,16, \mathrm{p}<.05$ ). However, in this case, the associated critical difference value indicated that not only was the arousal produced by the fast-rise stimulus significantly different from the control condition, but it was also significantly different from the arousal produced by the slow-rise stimulus. The slow-rise stimulus was, in turn, significantly different from the control condition. Thus, in summary, both types of auditory stimulation produced significant changes in the subject's sleeping EEG record but were different from 
one another only during sleep characterized by slowwave EEG activity.

\section{DISCUSSION}

In terms of characterizing sleep by the way in which an individual reacts to extrinsic stimulation, the present results appear to add another parameter to a growing catalogue. This parameter is stimulus onset. However, like certain other parameters, stimulus onset does not appear to be consistently effective over different types of sleep. That is, like frequency and the concomittant subjective attribute of loudness (LeVere et al, 1973, 1974), rise time or stimulus onset appear to be differentially effective only during sleep characterized by slow-wave electroencephalographic activity. During sleep characterized by non-REM fast-wave EEG activity, the onset character of an auditory stimulus seems to be quite unimportant in determining an individual's reaction to the stimulus.

While the above is a simple general summary of the empirical data, there are some details which are somewhat perplexing. For example, the results suggested that stimuli having an instantaneous onset appear to be more arousing than stimuli having a gradual onset. While it is possible to explain this result on the basis of a "startle effect," it must be remembered that the total amount of auditory energy and the total time that the auditory stimulus was present was considerably longer when the stimulus had a gradual onset and offset. This is so because each type of stimulus was presented for a duration of $15 \mathrm{sec}$ at its maximum intensity level and the gradual onset and offset of the slow-rise stimulus necessarily added considerable auditory energy to this stimulus. In terms of time alone, the slow-rise auditory stimulus was present for twice the duration of the fastrise stimulus. While it is, of course, possible to argue that the startle effect is a more significant parameter in producing changes in an individual's pattern of electroencephalographic activity than total auditory energy or stimulus duration, it is also possible to interpret the results in light of the character of sleep itself. That is, it is possible to suggest that sleep is a need state as indicated by data concerned with: (1) the rebound effect and (2) the necessity of increasing the amount of extrinsic stimulation required to maintain selective sleep deprivation (see Kales, 1969). Considering slowwave sleep as a need state which the individual may actively attempt to maintain, the decreased effectiveness of the slow-rise stimulus might be related to its gradual onset enabling intrinsic compensatory adjustments to maintain the sleep state. The rapid onset of the fast-rise stimulus, on the other hand, might disallow this sort of compensation to occur and thus precipitate a greater level of arousal in terms of cortical desynchronization. Whether or not this sort of explanation is more tenable than one relying on some sort of startle effect, if in fact the two explanations are necessarily incompatible, will remain to be determined by further research.

In a somewhat different vein, it is perhaps worthwhile to note that the present results are not directly predictable from research using awake individuals. Specificially, in both field studies (Kryter, Johnson, \& Young, Note 1) and laboratory investigations (Nixon, Von Gierke, \& Rosinger, 1969), the data indicate that auditory stimuli having a gradual onset are subjectively more annoying than auditory stimuli having a rapid onset. The present data, however, indicate quite the opposite. That is, stimuli having a gradual onset produce less cortical desynchronization. Thus, in terms of the waking data, it would seem necessary to assume that less annoying stimuli are more arousing during sleep, and vice versa-a somewhat bizarre position difficult to defend on simply common sense grounds. Given this, it is perhaps plausible to suggest that the subjective or psychological quality of an auditory stimulus may not contribute to an individual's electroencephalographic response during sleep, at least not during sleep characterized by slowwave patterns of electroencephalographic activity. This suggestion would be, of course, compatible not only with the present results but also with previous research by LeVere et al. $(1973,1974)$ which indicated that the psychological attribute of loudness was not a valid predictor of an individual's response during sleep characterized by slow-wave EEG activity. Taken together, then, the present data, as well as previously reported research, may lend some credance to the possibility that in some psychological sense certain types of sleep may be qualitatively distinct from wakefulness. The importance of this conjecture demands considerable empirical verification before accepting it without reservation, and it may be possible that one of the more fruitful methodologies to employ is a precise determination of how an individual responds to extrinsic stimulation during sleep.

\section{REFERENCE NOTE}

1. Kryter, K. D., Johnson, P. J., \& Young, J. R. Judgement tests of flyover noise from various aircraft. NASA Contract Report, CR-1635, 1969.

\section{REFERENCES}

Adrian, E. D., \& Matthews, B. H. C. Berger rhythm: Potential changes from the occipital lobes in man. Brain, 1934, 57, 355-385.

Amadeo, M., \& Shagass, C. Brief latency click-evoked potentials during waking and sleep in man. Psychophysiology, 1973, 10, 244-250.

FIRTH, H. Habituation during sleep. Psychophysiology, 1973, 10, 43-51.

Freemon, F. R. Sleep research. Springfield: Thomas, 1972.

Goodenough, D. R., Lewis, H. B., Shapiro, A., Jaret, L., \& Sleaser, I. Dream reporting following abrupt and 
gradual awakenings from different types of sleep. Journal of Personality and Social Psychology, 1965, 2, 170-179.

Johnson, L. V.. \& LuBIN, A. The orienting reflex during waking and sleeping. Electroencephalography and Clinical Neurophysiology, 1967, 22, 11-21.

JoNes. R. M. The problem of "depth" in the psychology of dreaming. Journal of Nervous and Mental Disease, 1964, 139. 507-515.

Kales, A. (Ed.) Sleep: Physiology and pathology. Philadelphia: Lippincott, 1969.

Kleitman, N. Sleep and wakefulness. Chicago: University of Chicago Press. 1963.

LeVere, T. E., Bartus, R. T., \& Hart, F. D. Arousal from sleep: Responsiveness to different auditory frequencies equated for loudness. Physiology and Behavior, 1973, 10. 53-57.

LeVere, T. E., Morlock, G. W., Thomas, L. P., \& HART, F. D. Arousal from sleep: The differential effect of frequencies equated for loudness. Physiology and Behavior, 1974, 12, 573-582.

LINDQuist, E. F. Design and analysis of experiments in psychology and education. Boston: The Riverside Press, 1956.

Mendel, M., \& Goldstein, R. Early component of the averaged electroencephalographic response to constant level clicks during all night sleep. Journal of Speech and Hearing Research, 1971, 14, 829-840.

Moruzzi. G., \& Magoun, H. W. Brain 'stem reticular formation and activation of the EEG. Electroencephalography and Clinical Neurophysiology, 1949, 1, 455-473.

Nixon. C. W., Von Gierke, H. E., \& Rosinger, G. Comparative annoyances of "approaching" versus "receding" sound sources. Journal of Acoustical Society of America, 1969, 45, 330.

Rechtschaffen, A., Hauri, P., \& Zeitlin, M. Auditory wakening thresholds in REM and NREM sleep stages.
Perceptual and Motor Skills. 1966, 22, 927-942.

Rechtschaffen, A., \& Kales, A. A Manual of Standardized Terminology. Techniques and Scoring System for Sleep Stages for Human Subjects. Washington, D.C: Public Health Service, U. S. Government Printing Office, 1968. Pp. 13.

Rheinberger, M., \& JASPER, H. H. Electrical activity of the cerebral cortex in the unanesthetized cat. American Journal of Physiology, 1937, 119, 186-196.

Sokolov. E. N. Neuronal models in orienting retlex. In M. A. B. Brazier (Ed.), The cerebral nervous system in behavior: Transactions of the 3rd conference. New York: Josiah Macy, Jr., Foundation, 1960.

Sokolov,.E. N., \& Paramonova, N. P. Progressive changes in the orienting reflex in man during the development of sleep inhibition. Pavlov Journal of Higher Nervous Activity, 1961, 11, 217-226.

Weitzman, E. B., \& Kreman, H. Auditory evoked responses during different stages of sleep in man. Electroencephalography and Clinical Neurophysiology, 1965, 18, 65-70.

Williams, H. L., Hammack, J. T., Daly, R. L., Dement, W. C., \& Lubin, A. Responses to auditory stimulation, sleep loss and the EEG stages of sleep. Electroencephalography and Clinical Neurophysiology, 1964, 16, 269-279.

Williams, H. L.. Tepas, D. I.. \& MoRlock, H. C. Evoked responses to clicks and electroencephalographic stages of sleep in man. Science, 1962, 138, 685-686.

Zung, W. W. K., \& Wilson, W. P. Response to auditory stimulation during sleep. Archives of General Psychiatry, $1961,4,40-44$.

(Received for publication September 4, 1975; revision accepted January 20, 1976.) 